\title{
The duty cycle in Functional Electrical Stimulation research. Part II: Duty cycle multiplicity and domain reporting
}

\author{
Matthew J. Taylor (1,2), Ché Fornusek (3), Andrew J. Ruys (1) \\ (1) Faculty of Engineering and IT, University of Sydney, Camperdown, Australia; (2) Charles \\ Perkins Centre, University of Sydney, Camperdown, Australia, (3) Faculty of Medicine and \\ Health, University of Sydney, Lidcombe, Australia \\ This article is distributed under the terms of the Creative Commons Attribution Noncommercial License (CC BY-NC 4.0) which permits \\ any noncommercial use, distribution, and reproduction in any medium, provided the original author(s) and source are credited.
}

\begin{abstract}
In part I of this review, we introduced the duty cycle as a fundamental parameter in controlling the effect of electrical stimulation pulse trains on muscle structural and functional properties with special emphasis on fatigue. Following on from a survey of the literature, we discuss here the relative ability of intermittent and continuous stimulation to fatigue muscle. In addition, pertinent literature is explored on a more deeper level, highlighting contentions regarding the duty cycle across studies. In response to literature inconsistencies, we propose frameworks upon which the duty cycle parameter may be specified. We present the idea of domain reporting for the duty cycle, and illustrate with practical examples. In addition we dig further into the literature and present a set of notations that have been used by different researchers to report the duty cycle. We also propose the idea of the duty cycle multiple, which together with domain reporting, will help researchers understand more precisely duty cycles of electrical stimulation. As a case study, we also show how the duty cycle has been looked at by researchers in the context of pressure sore attenuation in patients. Together with part I, it is hoped that the frameworks suggested provide a complete picture of how duty cycle has been discussed across the literature, and gives researchers a more trans-theoretical basis upon which they may report the duty cycle in their studies. This may also lead to a more precise specification of electrical stimulation protocols used in patients.
\end{abstract}

Key Words: functional electrical stimulation (FES), parameters, duty cycle reporting

Eur J Transl Myol 28 (4): 323-336, 2018

\begin{abstract}
Related to the concept of duty cycle are the terms "continuous" and "intermittent" stimulation. In simplest of terms, continuous stimulation is continuous, and intermittent stimulation has gaps where continuous would not. However, this is very open for interpretations. There is no standard definitions of what constitutes continuity versus intermittency in the muscle international literature. This is an issue that renders comparison of those studies that aim to compare continuous versus intermittent stimulation minimally comparable (e.g., compare some of the studies in table 2 of this review Part I $)^{1}$.
\end{abstract}

\section{Continuity and Intermittency - Which is More Fatiguing?}

Another more practical issue with comparing continuous versus intermittent stimulation is the conflicting literature, showing different opinions on the fatigue of muscles subjected to intermittent as opposed to, continuous stimulation. While it has been suggested that intermittent (or "cyclical") stimulation, with rest in between pulses is better in reducing fatigue than continuous stimulation by Krajl and colleagues, ${ }^{2}$ this is not always the case. Differences in continuous and intermittent stimulation patterns have been investigated by various authors such as Spriet \& colleagues. ${ }^{3}$ In their study of healthy quadriceps of 12 male subjects, Spriet et al. ${ }^{3}$ compared muscle responses between a continuous protocol of $102.4 \mathrm{~s}$ stimulation, with an intermittent protocol of the same time, but delivered at a duty cycle of 1:1 (1.6s ON, 1.6s OFF). Stimulation was performed at $20 \mathrm{~Hz}$. The authors found in the first $51.2 \mathrm{~s}$, that isometric force decreased more for the intermittent group (to $55 \%$ of initial isometric force), compared with the continuous group (to $80 \%$ of initial isometric force). This would suggest that fatigue was greater after stimulation with breaks. Yet other studies such as that of Duchateau and Hainaut ${ }^{4}$ suggest relaxation will quell 
Table 1. Postulate. Intermittent versus Continuous Stimulation

The fact that the relative ability of an intermittent protocol to be less fatiguing for a muscle compared to a continuous analogue, and vice versa for another muscle, warrants serious further investigation.

fatigue (Table 2, in review Part I) ${ }^{1}$. Although their study was on adductor pollicis, stimulation was provided over a similar time frame of $60 \mathrm{~s}$ under continuous stimulation. Therefore, the relative fatigue that intermittent and continuous stimulation may give is contested across the literature. This concept may be further concurred by examination of other studies. By the findings of Pournezam et al. ${ }^{5}$ and others (table 2, in part I $)^{1}$, it could be suggested that continuous electrical stimulation is much more fatiguing than intermittent, sequential stimulation. The authors do for example, argue that recovery is better for sequential as opposed to continuous stimulation. Their study also examined different types of sequential stimulation. They argued that 3-phase (i.e. RF, VL, VM) is better than 2-phase stimulation ( $\mathrm{RF}, \mathrm{VL}+\mathrm{VM})$, in the context of fatigue and recovery. However, other authors such as Duchateau and Hainaut ${ }^{4}$ argue that force decline is greater for intermittent, compared with continuous stimulation. There is no doubt that the methodologies employed by the authors were invariably different. In terms of muscles examined, one cannot directly compare quadriceps findings ${ }^{5}$ with that of adductor pollicis. ${ }^{4}$ Comparing these two papers provides evidence for the contention in the literature surrounding the relative effects of intermittent compared with continuous across different time domains. In light of these findings, an important postulate is put forward against the backdrop of the literature (Table 1).

\section{Further Contentions?}

The idea that a greater frequency of stimulation causes more fatigue may be contested by the findings of Matsunaga et al. ${ }^{6}$ The authors investigated duty cycles of $1 / 15,1 / 30$ and $1 / 60$, by providing stimulation for $4 \mathrm{~s}$ at the beginning of 60,120 and 240s (i.e., 4s ON, 56s OFF; 4s ON, 116s OFF; 4s ON, 236s OFF as inferred from their data). Experiments were conducted in healthy individuals $(n=20)$, and suffering with paraplegia $(n=$ 4). The paraplegic results are discussed here for illustrative purposes. Electrical stimulation was delivered as square monophasic waves, pulse width $200 \mu$ s. In the paraplegic group, six different protocols were used, and fatigue was recorded. Protocols involved stimulation at 20 or $100 \mathrm{~Hz}$ with duty cycles of $1 / 15$, $1 / 30$, or $1 / 60$. The authors used a strength decrement index (SDI) originally proposed by Clarke et al. ${ }^{7}$ to assess muscle fatigue, measuring quadriceps femoris torque with a KinCom isokinetic dynamometer. Interestingly, SDI was significantly greater after $20 \mathrm{~Hz}$ stimulation than $100 \mathrm{~Hz}$ ( $n=16$ tests). Moreover, SDI after $1 / 15$ was significantly greater than after $1 / 60$ (no significant difference between $1 / 15$ and 1/30, 1/30 and $1 / 60$ ) but by manual inspection of their data there is a downward trend. Krajl et al. ${ }^{2}$ put forward that fatigue can be reduced "...by using the lowest stimulation frequencies possible..." in their paper on ES standing for paraplegia. However, in a study Matsunaga et al. ${ }^{6}$ this would dictate otherwise. While context is important, such findings illustrate general differences in duty cycle and frequency relationships.

\section{Is the Duty Cycle Reported?}

The notion of continuous and intermittent stimulation differences is inextricably tied with the concept of choosing relevant $\mathrm{ON}$ and $\mathrm{OFF}$ times for exercise. This concept is important in all forms of FES exercise. However, in order to appreciate the true differences between continuous and intermittent stimulation, it is essential that the duty cycle is reported explicitly. The literature clearly indicates that duty cycle may not always be explicitly reported, or warrant in-depth analysis. Deley et al. ${ }^{8}$ for example, present a table of various studies and the duty cycles used by the authors. Papers listed include a collection of FES cycling, and muscle strengthening, papers. In all the FES cycling papers listed, they state that duty cycle is "not applicable". However, in most of the strengthening papers, duty cycles are presented. Indeed duty cycles are relevant, and are a cardinal feature of a train of impulses. ${ }^{8}$ Other than to facilitate the venture to have all FES protocols of a common nature, there are more important reasons as to why duty cycles should also be presented in all cycling papers as well.

\section{Standardized Definition. Proposal 1. Domain Reporting}

The relative ability of intermittent and continuous protocols to bestow fatigue upon muscles is thus conflicting, as illustrated from literature (Part I) ${ }^{1}$ Therefore, it follows that some theoretical guidance could facilitate the development of a more unified approach to discussing the relative degree of intermittency as compared with continuity of electrical stimuli. A fundamental approach is outlined in (Table 2 ), with relevant literature discussed. It is believed this theory here will help to provide a more homogeneous basis upon which such disparities could be studied. In addition, examples of duty cycles in terms of "domains" (i.e., over what time unit the duty cycle or stimulation is expressed), is also presented in Table 2. It is pertinent to note in the discussion of domains, that stimulation in the seconds domain is of particular interest for muscle 
Table 2. The Theory of Domain Reporting: A Fundamental Axiom for FES Protocols

\section{Statement:}

No electrical pulse train is purely continuous, as between pulses in the train there exists a series of interpulse intervals [e.g., Springer et al. ${ }^{9 *}$ ]. These intervals have to exist by the definition of frequency.

\section{Definition:}

An electrical pulse train is truly continuous if and only if the pulse width of the pulses in the pulse train multiplied by the frequency equates to a value of 1 [or any dimensionless multiple].

\section{Implication:}

For the sake of more commonality between FES protocols, there needs to be a standardized definition about what a "continuous" and what an "intermittent" pulse is. Moreover, the time domain of both interpulse intervals and pulses, as well as pulse train ON and OFF times should be reported in all FES protocols for the purposes of reporting total time $O N$ and total time OFF of stimulation. In addition, such reporting is essential if comparisons between continuous and intermittent protocols are going to be drawn in the context of fatigue and other relevant metabolic parameters. This follows on also, from the postulate of table 4.

\section{Relevant Literature - Examples of Continuous and Intermittent Comparisons:}

- Chasiotis et al. ${ }^{10}$

- Duchateau \& Hainaut 4 - compared "sustained" and intermittent.

- Bergström \& Hultman. ${ }^{11}$

*Springer \& colleagues discuss the "interphase interval", however is referenced here as is an example of literature which talks of smaller breaks in stimulation.

contractions, whereas milliseconds stimulation is related to nerve fibre recruitment.

\section{Pulse Width Reporting as Well?}

One element tied to theory of domain reporting is pulse width, which helps determine how long stimulation is turned on for in the microseconds or milliseconds domains. Hence, accurate reporting of pulse widths between study is requisite, and a fundamental part of the theory presented here. Pulse width is usually specified by FES protocols that investigate duty cycle, for example Deley et al. ${ }^{8}$ facilitating a researcher who would try and compute the total charge from this, with the relevant $\mathrm{ON} / \mathrm{OFF}$ time and current amplitude. However, a closer look at a publication by Bijak et al. ${ }^{19}$ could possibly make one draw skepticism to how pulse widths are reported. For example, in their work on FES standing and walking, the authors note that "...pulse duration 0.6 us +0.6 us..." was used, in the context of biphasic pulses. In light of the Bijak et al. ${ }^{19}$ reporting, one must question whether these values refer to the total or partial pulse width calculation. In other words, are the authors reporting the duration for half the pulse, or the total pulse?

\section{Issues with Duty Cycle Definition and Relevance}

In designing training protocols, it is imperative to understand how changing parameters of stimulation influences various aspects of a muscle contraction. This was highlighted in a paper by Packman-Braun ${ }^{20}$ in the late 1980s in her study of wrist muscles in hemiparesis, when she put forward:

"Some compromise between quality and quantity may be required to create the optimum training protocol" 20

The authors also ask: is it better to do more contractions, or less but

$$
\text { "... at a higher ... force output" } " 20
$$

Such a question highlights the importance of considering the training protocol in the design of a study, in addition to the parameter protocol implemented. Furthermore, Packman-Braun ${ }^{20}$ argue that if OFF time increases, there will be less muscle contractions, and that there is an inherent act of balancing when considering treatment.

\section{Critical Analysis - Definition and Varied Reporting}

A commonality that exists amongst the literature of ES is of course contention in both the best stimulation sequences to use, and definitional aspects of FES. This is why it is important to have a rigorous understanding of the definition of an electrical parameter. Duty cycle is often reported in different ways between authors, as highlighted in Table 4. Whether explicitly reported, or inferred, duty cycle differs between studies not just in terms of stimulation time ON compared with stimulation time OFF, but in what time domain this occurs. Indeed, the idea of time "domains" is the basis of the author's derivation of a system for reporting duty 
Table 3. Domains of Different Duty Cycles

\section{Milliseconds domain:}

- Laughman et al. ${ }^{12}$ : "10msec of sinusoidal output, 10-msec silent period"* Quadriceps, healthy humans. Seconds domain:

- Dreibati et al. ${ }^{13}: 60$ X 5s ON, 15s OFF for 20mins. Performed at 100, 50 \& $20 \mathrm{~Hz}$. Quadriceps, healthy humans.

- Gondin et al. ${ }^{14}$ : [A variety of papers presented by authors stipulating different duty cycles across studies].

- Gorgey et al. ${ }^{15}: 3 \mathrm{~s}$ ON, $3 \mathrm{~s}$ OFF, for $1 \mathrm{~min}$.

- Chou and Binder-Macleod ${ }^{16}:$ 1s ON, 9s OFF [inferred from their SCI protocol], their "testing trains"].

- Matsunaga et al. $.^{6}: 1 / 15$ (4s at start of 60s), 1/30 (4s at start of 120s), 1/60 (4s at start of 240s).

- Giannasi et al. ${ }^{17}: 12-19 \mathrm{~mA} 5 \mathrm{~s}$ ON 10s OFF 20mins. Stimulation of masseter and temporalis.

Minutes domain**:

- Minogue et al. ${ }^{18}: 4$ min stimulation, 4 min rest. However, the authors suggest that the 4 min were comprised of $5 \mathrm{~s}$ ON $5 \mathrm{~s}$ OFF periods.

* May be referring to an alternating current (AC) equivalent of an IPI.

**Not actually minutes domain, but seconds domain. However, placed here for discussion.

cycle in a common fashion between studies. To illustrate by example, the study by Minogue et al. ${ }^{18}$ reported stimulation as lasting 4 minutes both ON and OFF. However, studies by authors such as Dreibati et al. ${ }^{13}$ all utilize duty cycles which involve stimulation $\mathrm{ON}$ and OFF time in the domain of seconds. Therefore, the theorem presented in Table 3 could facilitate better understanding of what duty cycle is used by researchers if they adopt this paradigm. An example of a study where the reporting of duty cycle can be met with confusion is that of Song et al. ${ }^{21}$ The authors developed software to be used with an 8 channel stimulation system. They specified that duty cycle could be changed in the range $0-10 \mathrm{~s}$. There are questions which may come to mind when analyzing this statement. What exactly is being reported? Is this referring to $\mathrm{ON}$ or OFF times, or both ON and OFF times? Are ramp-ups/ramp-downs included? Also, a diagram of the software is presented by the authors which has a depiction of ramp up/ramp down, but it is unclear from the authors' work whether or not this is included in the "duty cycle". Such observations are just one example of contentions which stem from duty cycle literature. An account of literature inconsistencies is listed in Table 4, with important concepts related to duty cycle reporting variability. An issue which stems from the literature is that of notation, with different authors reporting duty cycles by use of different methods (Table 4). For example, in the review by Deley and colleagues, ${ }^{8}$ the authors present duty cycles from various researchers in terms of "dashes", such as 5-5 when reporting the work of Bélanger et al. ${ }^{22}$ Yet duty cycle is presented as fractions by Matsunaga et al. ${ }^{6}$ and percentages by Lieber and Kelly. ${ }^{23}$ Interconversions between such notations may be rendered confusing, preventing transparent comparison across studies. For example, Gondin et al. ${ }^{14}$ also reported duty cycles [of Herrero et al. ${ }^{24,25}$ ] as $2 / 1$. Herein these are discussed, using the Gondin et al. ${ }^{14}$ interpretation of the literature consulted. Confusion may arise when comparing this with for example, Matsunaga et al. ${ }^{6}$ duty cycles. These authors stipulate a duty cycle of for example, $4 \mathrm{~s}$ every 60 s (so $1 / 15$, or $6.667 \%$ ). If one was to convert Herrero et al. ${ }^{24,25} 2 / 1$ to a percentage this would imply that stimulation is on for $200 \%$ of the time. In parallel, in their study of denervated muscle, Ashley et al. ${ }^{26}$ used duty cycles of $1 / 2$ or $2 / 1$ depending on what stimulation pattern they were investigating. By their notation, these would be $1 \mathrm{~s}$ ON $2 \mathrm{~s}$ OFF or $2 \mathrm{~s}$ ON $1 \mathrm{~s}$ OFF. Hence, this "fractional notation" may not be all that convoluted. Yet, if comparing with a protocol which utilizes percentage notation, care must be taken not to misinterpret a $2 / 1$ duty cycle as a " $200 \%$ duty cycle" which has not realistic meaning. In this situation, what this definition means is that the duty cycle is defined relative to the OFF time. The ON time is $200 \%$, OFF time is $100 \%$, yet this sounds arduous. Finally, caution must be taken when comparing studies on a basis of their duty cycle findings, if one uses alternating current, the other pulsed current. An example of some confusion that may arise from the literature may be seen in examining the work of Moreno-Aranda and Seireg. ${ }^{33}$ Although the authors work was performed using AC stimulation of canine quadriceps, it does elucidate some important considerations when duty cycles are reported in alternating current studies. For example, the authors concluded that "maximum pull" for the dogs occurred at an "ON-OFF frequency" of $50 \mathrm{~Hz}$, and at a duty cycle of $50 \%$. This would suggest that "ON-OFF" and "duty cycle" are not synonymous. However, the authors do stipulate that the term "duty cycle" refers to: "Ratio of 
Table 4. Examples of Duty Cycle in The Literature and Contentions

Issue $\quad$ Example from Literature

\#1: Different notation used Fractional notation. Matsunaga et al. ${ }^{6}$ report "periods" of stimulation as a to denote duty cycle

fraction of a certain period. E.g. $4 \mathrm{~s}$ at beginning of $60 \mathrm{~s}$ period $=1 / 15$.

Descriptive notation. Minogue et al. ${ }^{18}$ report "... bouts of intermittent NMES, each bout lasting 4 minutes, with a rest interval of 4 minutes between each bout". [Yet stimulation is delivered in the seconds domain within these minutes, also].

Descriptive notation. Currier and $\mathrm{Mann}^{27} *$ report a long-winded description of a $15 \mathrm{~s}$ ON (5s ramp, 10s peak intensity), 50s OFF protocol

ON/OFF notation. Carmick ${ }^{28}$ report "...10 seconds on and 25 seconds off...", for example.

Percentage notation. Lieber and Kelly ${ }^{24 * *}$ report " $50 \%$ duty cycle (5 s stimulation, $5 \mathrm{~s}$ rest)***"

Dash notation. Deley et al. ${ }^{8}$ presents duty cycles from a variety of literature. One such example is " $5-5$ " from Bélanger and colleagues,${ }^{22}$ who report in their study “...5-sec on/5-sec OFF duty cycle...". Another example is their reporting of Crameri et al.'s ${ }^{29}$ duty cycle as " 4 ".

\section{\#2: Duty cycles are often See Table 3.}

\section{reported but the}

domains $* * * *$ are different

across studies.

\section{\#3: Duty cycles}

sometimes $* * * * *$ include

ramp up and ramp down

times, sometimes do not.

\section{\#4: Sometimes}

information may be

missing from the reported

e.g., Baker et al. ${ }^{30}$ stipulates that ramps not usually included in duty cycle time.

duty cycle (explicitly or

implicitly reported DC's),

hence it is hard to know

what the exact duty cycle

is.

*[Regarding: Currier and $\mathrm{Mann}^{27}$ ]: "Tetanic isometric muscular contractions lasted for 15 seconds, during which time the current was surged so that peak intensity was reached after 5 seconds and sustained for 10 seconds. At the end of the 15 -second current stimulating period, the intensity abruptly declined and was followed by a 50 -second rest before the next stimulating period (isometric producing repetition)." (Currier and Mann, 1983).

**[Regarding: Lieber and Kelly $\left.{ }^{23}\right]$ : The authors report: $50 \%=5 \mathrm{~s}$ ON $5 \mathrm{~s}$ OFF, $70 \%=5 \mathrm{~s}$ ON $2 \mathrm{~s}$ OFF. Yet $5 / 7 * 100=71.43 \%$ ON...so they may be roughly accurate. A good idea would be for the authors to rather rename their study one which focuses on the effects of different relaxation time intervals, to be more precise.

$* * *$ This is also a modified ON/OFF notation as well. E.g. "...stimulation....rest..." ${ }^{23}$.

****The author of this paper proposes notation for duty cycle domains.

*****Janssen and Pringle ${ }^{32}$ mention 5 s rest...how long are contractions?

time ON and OFF of the stimulation signal". ${ }^{33}$ While all the literature has aimed at advancing our understanding of different stimulation regimes for FES, it is clear that a more common method for reporting duty cycle is desirable.

\section{Standardized Definition. Proposal 2: Duty Cycle and Duty Cycle Multiplicity}

Duty Cycle Definition

Let the duty cycle be defined in the following unambiguous manner:

Duty cycle $=\mathrm{X} s$ ON, Ys OFF

With a RU of $\mathrm{Z} s$ and RD of $\mathrm{W} s$
Where:

- X $s$ ON denotes stimulation which is on $(\mathrm{ON})$ for $\mathrm{X} s$,

- Ys OFF denotes stimulation which is off (OFF) for $\mathrm{Y}$ $s$,

- RU of Z $s$ denotes a ramp-up (RU) time of Z $s$,

- RD of W $s$ denotes a ramp-down (RD) time of W $s$.

This notation of ON and OFF is used extensively to describe, and is derived from, the literature presented in part I of this review. ${ }^{1}$ The form above is one with minimal ambiguity, allowing for full information of the duty cycle to be described. In a practical sense, this is illustrated with some examples: 
- The duty cycle is $2 s \mathrm{ON} 3 \mathrm{~s} \mathrm{OFF}(\mathrm{RU}=\mathrm{RD}=1 \mathrm{~s})$.

- The duty cycle is $1 s$ ON 5s OFF ( $R U=2 s, R D=$ $0.5 \mathrm{~s})$.

In the second example, there is a greater ramp-up. In the first, ramp-up and ramp-down are the same.

Duty Cycle Multiple - $T_{f}$

What is more important is the concept of ambiguity, as discussed previously. For example, a 2:10 and 1:5 both are a 1:5 duty cycle, but with different multiples of 2 , and 1, respectively (i.e., $1: 5=1: 5 \times 1,2: 10=1.5 \times 2$ ). Let this be the duty cycle multiple $\left(\boldsymbol{T}_{f}\right)$. It follows from the above example, that the duty cycles may alternatively be expressed in a format with a fundamental duty cycle (FDC) and corresponding $T_{f}$ as such:

$$
\text { 1:5 }\left(T_{f}=2\right), 1: 5\left(T_{f}=1\right) \text { respectively. }
$$

Wherein the term "fundamental" could be thought of being inspired by engineering and physics concepts related to "fundamental frequency". While of course this, with this definition, it may be seem to be "playing with semantics", indeed specification of the duty cycle multiple is useful in the design of experiments when comparisons of several duty cycles are being made, to see which ones have the same percentage ON and OFF time (an FDC with any multiple having the same percentage of time spent $\mathrm{ON}$ and $\mathrm{OFF}$ ). For example, Baksay, ${ }^{34}$ in his thesis examining ON and OFF times for thigh stimulation, used duty cycles of 5:15, 10:30 and 15:45. By the notation presented, these would correspond to an FDC of 5:15, and $T_{f}$ values of 1,2 and 3 , respectively. This reference is just one example of literature where the same duty cycle is used, with different multiples (e.g., Gentz and Moore ${ }^{35}$ from Part $\left.\mathrm{I}^{1}\right)$. There are also other studies where only one of ON or OFF is permuted (see Smit and colleagues below, for example). ${ }^{48}$ Therefore, the use of the $T_{f}$ notation can be modified accordingly. For example, in another work we have used the duty cycles of 1:3, 2:3 and 3:3. ${ }^{36}$ This could be represented as:

$$
1: 3\left(T_{f, \text { oN }}=1,2,3\right),
$$

to denote the set of duty cycles tested. In that work, we also tested duty cycles where OFF time was doubled and trebled and both ON and OFF also. Therefore, to comprehensively represent the set of seven duty cycles tested, a set notation could be adopted:

$$
1: 3\left(T_{f} ; T_{f, \text { ON }} ; T_{f, \text { OFF }}\right)=(1,2,3 ; 1,2,3 ; 1,2,3) .
$$

In this case we have a special example of when permutations are all the same so this could be further contracted as such:

$$
1: 3\left(T_{f}=T_{f, \text { ON }}=T_{f, \text { OFF }}=1,2,3\right) .
$$

\section{Stimulation Ramp Times}

While ON and OFF times are important in specifying a duty cycle, so too are ramps. Ramp times are times taken to turn stimulation up to a desired value, or to turn down from that value to baseline (in this discussion, the focus is current amplitude). However, increase of pulse width has also been described, for example in Benton and Montgomery. ${ }^{37}$ Respectively, these are known as the "ramp-up" and "ramp-down" times. Hence, as they are related to ON and OFF times, they warrant mention in any "duty cycle" discussion. Moreover, they have an important influence on the contractile manifestation of muscle movement that occurs due to an applied stimulus. It has been discussed in literature on FESwalking by Bijak and colleagues, that ramp times are essential for providing "...natural movement..." as opposed to sudden ON/OFF stimulation, ${ }^{19}$ that would lead to considerable sporadicity in movements. Ramps are often reported in cycling studies, such as Sijobert et al. ${ }^{38}$ Another matter of contention to stem from the literature relates to the inclusion, or exclusion, or rampup times in the duty cycle numbers. Baker et al. ${ }^{30}$ stipulate that usually ramp up and ramp down are not included in the duty cycle definition. Yet, in the same publication, the authors discuss how there is an inherent variability with different stimulators, in the context of how ramp up and ramp down are included in ON and OFF definitions. The authors stipulate that OFF time should be determined by the "plateau ON time". ${ }^{30}$ In an early paper by Baker et al. ${ }^{39}$ the authors stipulate that "...the stimulation cycle lasts for seven seconds, followed by a 10-second rest interval". They then say that in the ON time, stimulation increases over $3 \mathrm{~s}$ to the max (exponentially), then is held there for $4 \mathrm{~s}$. Therefore, ramp times may be reported in various ways across studies. Not only does the inclusion of ramps in ON/OFF times vary across FES equipment, but also between various research groups. Packman-Braun for example, ${ }^{20}$ defined $7 \mathrm{~s}$ ON time as being comprised of; 2-s ramp-up, 5-s contraction in their study of wrist extensors. Duty cycles were defined relative to $5 \mathrm{~s}$ ON time. ${ }^{20}$ This variation in definitions of $\mathrm{ON}$ and OFF that exist between stimulators and authors render cross-study comparison difficult. This also suggests that a superficial reading of papers which examine duty cycle can lead the reader to make false conclusions, unless they truly understand how "duty cycle" is defined, and whether or not ramp-up and ramp-down times are included in its' definition. The precise reporting of ramp-up times is also an important consideration when discussing duty cycles, as such timing may change over time according to the treatment purpose. Carmick, for example ${ }^{40}$ comments on how ON times can be reduced over time, for children with cerebral palsy after they have become comfortable with the electrical stimulation used. Carmick also changed ramp-up times from $8 \mathrm{~s}$ to $2 s$ in her study of ES for cerebral palsy. ${ }^{28}$ Similarly, Carmick also changed ramp-up from $8 \mathrm{~s}$ to $2 \mathrm{~s},{ }^{41}$ in accordance with comfort, in her study on upper limb ES in cerebral palsy. Carmick focussed on lower limb..$^{28}$ In the 1993a study, ${ }^{28}$ the author also noted that when ES was required to control gait, $0.5 \mathrm{~s}$ ramp-up was utilized. 
Table 5. Duty Cycles and Pressure Sore Attenuation

- Baker et al. ${ }^{45 *}$ conducted a study on individuals with spinal cord injury to examine the effects of three different stimulation patterns on wound healing of pressure sores. They argued asymmetrical biphasic is the best type to use. In all cases, a 7:7 was utilized. This illustrates a 1:1 duty cycle can be used to attenuate pressure ulcers.

- Bogie et al. ${ }^{\mathbf{4 6}}$ also investigated use of ES for pressure sores. The authors report a case study of one participant (C4 SCI) with implantable percutaneous electrodes. The stimulation was of the glutei, $20 \mathrm{~Hz}$, 8 s ON 4s OFF (initially), followed by $20 \mathrm{~Hz} 15$ s ON 15 s OFF (second phase - a "dynamic" protocol in concordance with what the authors stipulate is important to prevent tissue wastage. The authors discovered that upon using ES, tissue oxygenation of the ischial region decreased.

- In a larger trial, Van Londen et al. ${ }^{47 * *, * * *}$ also investigated use of surface ES for pressure sores, in 13 individuals with SCI. They compared stimulation delivered to each glutei at once ("simultaneously") with that delivered to each gluteal in succession ("alternately"). The simultaneous protocol was $0.5 \mathrm{~s}$ ON $15 \mathrm{~s}$ OFF for both glutei at the same time, whereas the alternating protocol was given as $0.5 \mathrm{~s}$ ON $15 \mathrm{~s}$ OFF with one glutei by itself followed by the other. The authors concluded that the various pressures measured did not differ between alternating and simultaneous protocols.

- $\underline{\text { Smit et al. }}$. $^{\mathbf{2}}$ compared duty cycles of 1:1, 1:4 (1s domain) for $3 \mathrm{~h}$ of stimulation of hamstrings and gluteals in SCI. 1:4 was found to have a greater decrease in ischial tuberosity pressure, and less fatigue than 1:1.

\section{Salient Remarks:}

- *[Regarding: Baker et al., 1996]: Asymmetrical pulses were 100us, 50Hz, 7:7. Symmetrical pulses were 300us, $50 \mathrm{~Hz}, 7: 7$. Care must be taken in interpretation of the results, as the pulse width of the asymmetrical pulse was one-third that of the symmetrical pulse. Also tested were microcurrent pulses 10us, $1 \mathrm{~Hz}, 7: 7$. By the proposed notation of this paper, this is a 1:1 duty cycle, with $T_{f}=7$.

- ** The authors state in their crossover study design that 15 mins rest was given between stimulation using another protocol. Yet, other authors usually give $48 \mathrm{hrs}\left(\mathrm{e} . \mathrm{g}\right.$., Baksay ${ }^{34}$ ) in between stimulation sessions for example. One could argue that the works of Van Londen et al. (2008) may have disregarded this fatigue.

- ***[Regarding: Van Londen et al., 2008]: One could argue that there would have possibly been some differences should this protocol be repeated with some further rigour. For example, the alternating protocol involved $0.5 \mathrm{~s} \mathrm{ON} 15 \mathrm{~s}$ OFF on one glutei, then $0.5 \mathrm{~s}$ ON $15 \mathrm{~s}$ OFF for the other for 60 total repetitions ( $31 \mathrm{mins}$ total, with $15.5 \mathrm{mins}$ each glutei). The simultaneous protocol involved $0.5 \mathrm{~s}$ ON $15 \mathrm{~s}$ OFF on both glutei for 120 total repetitions (31 mins total, with $31 \mathrm{mins}$ each glutei). It is clear from these simple calculations that the simultaneous protocol involved stimulation for each glutei twice the time of the alternating protocol. The simultaneous protocol could have been done for $15.5 \mathrm{mins}$ total to ensure comparable stimulation times between the two protocols.

In addition, stimulation was given initially at $10 \mathrm{~s}$ ON $25 \mathrm{~s}$ OFF (i.e. 1:2.5), then changed to $15 \mathrm{~s}$ ON $15 \mathrm{~s}$ OFF (i.e. 1:1) when the patient gained comfort with the protocol. Long ramp-up times of $12 \mathrm{~s}$ have also been reported, in stimulation contexts where current as high as $300 \mathrm{~mA}$ has been used such as was the case in a study by Janssen and Pringle. ${ }^{32}$ Ramp-down times have also been included as a variable in the $\mathrm{ON}$ timing for stimulation. On the basis of prior art [namely, Jubeau et al. ${ }^{42}$ and Lyons et al. $^{43}$ ], Aldayel et al. $^{44}$ defined the ramp-up and ramp-down characteristics of their stimulation protocol for studying the differential effects of alternating EMS and pulsed EMS. The authors note that they used a $25 \%$ duty cycle ( 5 s ON, 15 s OFF). The 5s ON was inclusive of a 1s ramp-up, and a 1-s rampdown. Hence, the total time at maximum stimulation in each $5 \mathrm{~s}$ session was $3 \mathrm{~s}$. It is thus apparent that the true meaning of "ON" time is somewhat obfuscated in light of the various differences in study designs which choose to either include or exclude ramp-up/down times from the $\mathrm{ON}$ time definition. It is important that precision is taken into serious consideration, in light of studies such as Bijak et al. ${ }^{19}$ which argue that ramp-up times in FES walking for example, are dependent on the mass of the patient. The authors argue that ramp time is a pertinent issue in the context of optimization of FES standing. They stipulate that fast ramps (i.e., 0.2s) are more suitable for patients with a heavy weight, while longer 
ramps (i.e., 0.4s) are better in patients with a smaller weight. $^{19}$

\section{Case Studies in Light of the Proposed Frameworks}

The duty cycle is an important parameter to modulate when controlling FES-evoked exercises. Here we illustrate the use of the novel proposed conceptual ideas, when describing some of the literature regarding two distinct examples: stimulation for pressure sores, and FES-cycling.

\section{Case Study 1: Pressure Sores}

While the duty cycle has been examined in the context of fatigue and differential metabolic effects (Table 2, in review Part I) ${ }^{1}$, it has also been studied in the context of pressure sores. Relevant results are here discussed in Table 5. As previously reported, ${ }^{1}$ these studies are summarised in chronological order. Examining the stimulation parameters of Smit et al. ${ }^{48}$ more closely, the authors used duty cycles of $1: 1$ and 1:4. As these were in time multiples of $1 \mathrm{~s}$, by the $T_{f}$ notation, this can be represented as having performed stimulation at 1:1 $\left(T_{f}\right.$, $O F F=1,4)$. This allows focus to be drawn on the ON or OFF (in this case, OFF) time that is being permuted. As seen in Table 2 of Part $\mathrm{I},{ }^{1}$ the domain of stimulation has also been annotated.

\section{Case Study 2: Duty Cycles and FES Cycling}

There are many types of FES exercise available, such as FES-cycling, FES-rowing and FES-exoskeletons. ${ }^{36}$ However, FES-cycling is widely reported across the literature. Therefore, insights into duty cycle reporting may be derived from FES cycling research. In the context of ES cycling, stimulation is usually reported for muscle groups in terms of the angles of the cadence through which certain groups are turned on (e.g., Berkelmans). ${ }^{49}$ By this observation, it seems that duty cycle need not be reported - angles of the cadence clearly can be used to calculate how long a muscle is switched on per revolution. However, in light of recent work by Fornusek et al., ${ }^{50}$ it would be helpful to the field of FES if these ON and OFF values could be converted to percentages or fractions (such as, e.g, Matsunaga et al. ${ }^{6}$ ), to allow for comparison of the outcomes of cycling studies with isometric studies. For example, Fornusek et al. ${ }^{50}$ compared isometric and concentric exercise. Furthermore, if there were a common way of reporting duty cycles then perhaps these results could be compared more easily with other studies, and comparisons between cycling (rpm indicative of duty cycle) and isometric ( $s$ ON $s$ OFF indicative of duty cycle) could be made.

\section{Conclusive Summary}

The duty cycle is an important electrical stimulation parameter insofar that the degree of continuity or intermittency as defined by it can have differential effects on muscle characteristics, including fatigue. In Part I of this review, ${ }^{1}$ we showed that its' definition differs across literature, and we presented a survey of the pulsed current literature with critical commentary. This work can hopefully be used by researchers who are conducting studies examining how duty cycle affects fatigue, through providing an overview of how others have attempted to understand this parameter. In addition to the review presented, meta-analyses could be further conducted in the future which look at duty cycle. A meta-analysis could stratify electrical stimulation studies on a basis of: a) acute vs chronic studies (i.e. does the study look at bouts of 10 contractions etc., or does it look at an intervention over time for a few weeks?), and/or; b) duty-cycle-focused vs non-dutycycle-focused (i.e. does the study aim to look at the effects of different duty cycles, or is this a secondary outcome of the study?). The papers presented highlight issues which need to be addressed in terms of duty cycle reporting, which could provide the basis for such a review. In 1988, Packman-Braun ${ }^{20}$ argued that there needs to be "clarification" of using duty cycles with desirable fatigue responses, in order to allow, among other things, that "...the various therapeutic effects of treatment with FES will not be undermined by a poor choice of stimulation characteristics". ${ }^{20}$ While authors such as Baker have done tremendous work in presenting therapeutic indications with duty cycle in consideration, ${ }^{30}$ this review has shown there is still more fundamental work required in terms of precision in study reporting. ${ }^{1}$ In part II, we proposed the concepts of domain reporting, and duty cycle notation. ${ }^{51}$ This was done in the backdrop of literature that variably reports on duty cycle. Therefore, we hope these concepts will broaden the FES community's understanding of duty cycle reporting while also becoming aware of its' significance. Perhaps one step in the right direction in highlighting the importance of this parameter was a recent patent by Ranu (USPTO 9,643,010 B2) which details a system focused on the duty cycle. ${ }^{52}$ While frequency, pulse width and amplitude are more welldescribed across the literature, it is evident that focus on duty cycle is required for a complete picture of the stimulation specification when muscle is subjected to FES. These two reviews have provided a substantial overview of the literature pertaining to the duty cycle in Functional Electrical Stimulation applications. However, future work would also be well-guided to examine the influence of duty cycle modulation in situations where stimulation is delivered for multiple hours during the day. This has been explored for example in the work of researchers examining latissimus dorsi stimulation following cardiomyoplasty, ${ }^{52-57}$ where it has been suggested to provide periods of rest as opposed to continuous daily stimulation. In addition, use of FES in healthy and weakened/pathological muscles requires different training protocols. Therefore, consideration of the duty cycle across various muscle conditons would also be of immense interest in clinical FES uses, in particular for 
managements of transiently and permanent denervated muscles. ${ }^{58-84}$ New rehabilitation strategies developed in Vienna and the commercial availability of a pourpose developed electrical stimulator (Stimulette $\operatorname{den} 2 \mathrm{x})^{85}$ and large electrodes open new hopes to spread worl-wide managements to recover long-term permanent denervated human muscles by Home-based FES. ${ }^{61-72}$ Furthermore, we are confident that our suggestions will be followed in the training of those thoracic-level SCI persons after their enrollment in the future studies on FES intrethecal approaches. ${ }^{87-98}$ Taken together, these applications show the clinical importance of understanding differences in continuous and intermittent stimulation. Therefore, further exploration of duty cycle modulation in FES exercise across different patient cohorts is justified and mandatory.

\section{List of acronyms}

$\mathrm{AC}$ - alternating current

ES - electrical stimulation

EMS - electrical muscle stimulation

FDC - Fundamental Duty Cycle

FES - Functional Electrical Stimulation

$\mathrm{Hz}-\mathrm{Hertz}$

IPI - interpulse interval

$\mathrm{RF}$ - rectus femoris

SDI - strength decrement index

$T_{f}$ - Duty Cycle Multiple

$\mathrm{VL}$ - vastus lateralis

$\mathrm{VM}$ - vastus medialis

\section{Author's contributions}

MJT drafted the manuscript and was responsible for literature review and critical synthesis and analysis of literature, $\mathrm{CF}$ and AJR gave feedback on the manuscript.

\section{Acknowledgments}

MJT is a PhD student at the University of Sydney, Australia. He wish to acknowledge Professor Ugo Carraro, M.D. for his advice on structuring of the manuscript.

Funding: None.

\section{Conflict of Interest}

The authors declare no conflicts of interests.

\section{Ethical Publication Statement}

We confirm that we have read the Journal's position on issues involved in ethical publication and affirm that this report is consistent with those guidelines.

\section{Corresponding Author}

Mr. Matthew James Taylor, Level 3 East, Charles Perkins Centre D17, The University of Sydney, NSW, 2006, Australia. Phone: + 6128627 0521. Mobile: + 61 478033 859. Email: matthew.j.taylor@ sydney.edu.au

E-mail of co-authors
Ché Fornusek: che.fornusek@sydney.edu.au Andrew J. Ruys: andrew.ruys@sydney.edu.au

\section{References}

1. Taylor MJ, Fornusek C, Ruys AJ. Reporting for Duty: The duty cycle in Functional Electrical Stimulation (FES) research - Part I: Critical commentaries of the literature. Eur J Transl Myol 2018;28: in press.

2. Krajl A, Bajd T, Turk R, Benko H. Posture switching for prolonging functional electrical stimulation standing in paraplegic patients. Paraplegia 1986;24:221-30.

3. Spriet LL, Soderlund K, Hultman E. Energy cost and metabolic regulation during intermittent and continuous tetanic contractions in human skeletal muscle. Can J Physiol Pharmacol 1988;66:134-9.

4. Duchateau J, Hainaut K. Electrical and mechanical failures during sustained and intermittent contractions in humans. J Appl Physiol 1985;58:942-7.

5. Pournezam M, Andrews BJ, Baxendale RH, et al. Reduction of muscle fatigue in man by cyclical stimulation. J Biomed Eng 1988;10:196-200.

6. Matsunaga T, Shimada Y, Sato K. Muscle fatigue from intermittent stimulation with low and high frequency electrical pulses. Arch Phys Med Rehabil 1999;80:48-53.

7. Clarke HH, Shay CT, Mathews DK. Strength decrement of elbow flexor muscles following exhaustive exercise. Arch Phys Med Rehabil 1954;35:560-7.

8. Deley G, Denuziller J, Babault N. Functional electrical stimulation: cardiorespiratory adaptations and applications for training in paraplegia. Sports Med 2015;45:71-82.

9. Springer S, Bruan-Benyamin O, Abraham-Shitreet $\mathrm{C}$, et al. The effect of electrode placement and interphase interval on force production during stimulation of the dorsiflexor muscles. Artif Organs 2014;38:E142-E146.

10. Chasiotis D, Bergström M, Hultman E. ATP utilization and force during intermittent and continuous muscle contraction. J Appl Physiol 1987;63:167-74.

11. Bergström M, Hultman E. Energy cost and fatigue during intermittent electrical stimulation of human skeletal muscle. J Appl Physiol 1988;65:1500-5.

12. Laughman RK, Youdas JW, Garrett TR, Chao EYS. Strength changes in the normal quadriceps 
femoris muscle as a result of electrical stimulation. Phys Ther 1983;63:494-9.

13. Dreibati B, Lavet C, Pinti A, Poumarat G. Influence of electrical stimulation frequency on skeletal muscle force and fatigue. [Influence de la fréquence en stimulation électrique sur la force et la fatigue musculaire]. Ann Phys Rehabil Med 2010;53:266277. [Abstract and paper in English and French].

14. Gondin J, Cozzone PJ, Bendahan D. Is highfrequency neuromuscular electrical stimulation a suitable tool for muscle performance improvement in both healthy humans and athletes? Eur J Appl Physiol 2011;111:2473-87.

15. Gorgey, AS, Black, CD, Elder, CP, Dudley, GA. Effects of electrical stimulation parameters on fatigue in skeletal muscle. J Orthop Sports Phys Ther 2009;39:684-92.

16. Chou L-W, Binder-Macleod SA. The effects of stimulation frequency and fatigue on the forceintensity relationship for human skeletal muscle. Clin Neurophysiol 2007;118:1387-96.

17. Giannasi LC, Matsui MY, Freitas SRB, et al. Effects of neuromuscular electrical stimulation on the masticatory muscles and physiologic sleep variables in adults with cerebral palsy: a novel therapeutic approach. PLoS ONE 2015;10(8):e0128959.

18. Minogue CM, Caulfield BM, Lowery MM. Whole body oxygen uptake and evoked knee torque in response to low frequency electrical stimulation of the quadriceps muscles: $\mathrm{VoO} 2$ frequency response to NMES. J Neuroeng Rehabil 2013;10:63.

19. Bijak M, Rakos M, Hofer C, et al. Stimulation parameter optimization for FES supported standing up and walking in SCI patients. Artif Organs 2005;29:220-3.

20. Packman-Braun R. Relationship between functional electrical stimulation duty cycle and fatigue in wrist extensor muscles of patients with hemiparesis. Phys Ther 1988;68:51-6.

21. Song T, Yi JH, Khang G, Choi KW. An 8-channel stimulation pattern generator. IFMBE Proceedings, MEDICON 2001, 12-15 June 2001, Pula, Croatia.

22. Bélanger $M$, Stein RB, Wheeler GD, et al. Electrical stimulation: Can it increase muscle strength and reverse osteopenia in spinal cord injured individuals? Arch Phys Med Rehabil 2000;81:1090-8.
23. Lieber RL, Kelly M. Torque history of electrically stimulated human quadriceps: Implications for stimulation therapy. J Orthop Res 1993;11:131-41.

24. Herrero AJ, Martin J, Martin T, et al. Short-term effect of plyometrics and strength training with and without superimposed electrical stimulation on muscle strength and anaerobic performance: A randomized controlled trial Part II. J Strength Cond Res 2010a;24:1616-22.

25. Herrero AJ, Martin J, Martin T, et al. Short-term effect of strength training with and without superimposed electrical stimulation on muscle strength and anaerobic performance. A randomized controlled trial. Part I. J Strength Cond Res 2010;24:1609-15.

26. Ashley Z, Sutherland H, Russold MF, et al. Therapeutic stimulation of denervated muscles: The influence of pattern. Muscle Nerve 2008;38:875-86.

27. Currier DP, Mann R. Muscular strength development by electrical stimulation in healthy individuals. Phys Ther 1983;63:915-21.

28. Carmick J. Clinical use of neuromuscular electrical stimulation for children with cerebral palsy, Part 1: Lower extremity. Phys Ther 1993;73:505-13.

29. Crameri RM, Cooper P, Sinclair PJ, et al. Effect of load during electrical stimulation training in spinal cord injury. Muscle Nerve 2004;29:104-11.

30. Baker LL, Wederich CL, McNeal DR, et al. Neuro Muscular Electrical Stimulation - A Practical Guide (4thed). Rehabilitation Engineering Program, Los Amigos Research \& Education Institute, Rancho Los Amigos National Rehabilitation Centre, United States of America; 2000.

31. Soo C-L, Currier DP, Threlkeld AJ. Augmenting voluntary torque of healthy muscle by optimization of electrical stimulation. Phys Ther 1988;68:333-7.

32. Janssen TWJ, Pringle DD. Effects of modified electrical stimulation-induced leg cycle ergometer training for individuals with spinal cord injury. J Rehabil Res Dev 2008;5:819-30.

33. Moreno-Aranda J, Seireg A. Force response to electrical stimulation of canine skeletal muscles. J Biomech 1981;14:595-9.

34. Baksay SA. The effect of various stimulus ON:OFF times of a 1:3 duty cycle on quadriceps femoris muscle fatigue. Master of Science in Physical Therapy Thesis, School of Physical Therapy, Pacific University, Forest Grove, Oregon, United States of America; 1993. 
35. Gentz L, Moore C. Effect of duty cycle on fatigue during stimulation of the quadriceps muscle. Phys Ther 1988;834-834 [Abstract R-287].

36. Taylor MJ, Fornusek C, de Chazal P, Ruys AJ. "All talk no torque"- A novel set of metrics to quantify muscle fatigue through isometric dynamometry in Functional Electrical Stimulation (FES) muscle studies. 4th International Conference on Mechanical Engineering Research (ICMER2017). Kuantan, Malaysia, Aug 1-2, 2017. IOP Conference Series: Materials Science and Engineering, 257, 012018.

37. Benton LA, Montgomery J. Functional electrical stimulation: A practical clinical guide (2nd ed.) Professional Staff Association, Rancho Los Amigos Rehabilitation Engineering Center, Rancho Los Amigos Hospital; Downey, CA 198131-52.

38. Sijobert B, Fattal C, Daubigney A, Azevedo-Coste C. Participation to the first Cybathlon: an overview of the FREEWHEELS team FES-cycling solution. Eur J Transl Myol 2017;27(4):7120. doi: 10.4081/ejtm.2017.7120. eCollection 2017 Dec 5.

39. Baker LL, Yeh C, Wilson D, Water RL. Electrical stimulation of wrist and fingers for hemiplegic patients. Phys Ther 1979;59:1495-9.

40. Carmick J. Guidelines for the clinical application of neuromuscular electrical stimulation (NMES) for children with cerebral palsy. Pediatr Phys Ther 1997;9:128-36.

41. Carmick J. Clinical use of neuromuscular electrical stimulation for children with cerebral palsy, Part 2: Upper extremity. Phys Ther 1993;73:514-27.

42. Jubeau M, Sartorio A, Marinone PG, et al. Comparison between voluntary and stimulated contractions of the quadriceps femoris for growth hormone response and muscle damage. J Appl Physiol 2008;104:75-81.

43. Lyons CL, Robb JB, Irrgang JJ, Fitzgerald GK. Differences in quadriceps femoris muscle torque when using a clinical electrical stimulator versus a portable electrical stimulator. Phys Ther 2005;85:44-51.

44. Aldayel A, Muthalib M, Jubeau M, et al. Muscle oxygenation of vastus lateralis and medialis muscles during alternating and pulsed current electrical stimulation. Eur J Appl Physiol 2011;111:779-87.

45. Baker LL, Rubayi S, Villar F, Demuth SK. Effect of electrical stimulation waveform on healing of ulcers in human beings with spinal cord injury. Wound Repair Regen 1996;4:21-8.

46. Bogie KM, Wang X, Triolo RJ. Long-term prevention of pressure ulcers in high-risk patients: a single case study of the use of gluteal neuromuscular electric stimulation. Arch Phys Med Rehabil 2006;87:585-91.

47. Van Londen A, Herwegh M, van der Zee $\mathrm{CH}$, et al. The effect of surface electric stimulation of the gluteal muscles on the interface pressure in seated people with spinal cord injury. Arch Phys Med Rehabil 2008;89:1724-32.

48. Smit CAJ, Legemate KJA, de Koning A, et al. Prolonged electrical stimulation-induced gluteal and hamstring muscle activation and sitting pressure in spinal cord injury: Effect of duty cycle. J Rehabil Res Dev 2013;50:1035-45.

49. Berkelmans R. FES cycling. J Automatic Control 2008;18:73-6.

50. Fornusek C, Gwinn TH, Heard R. Cardiorespiratory responses during functional electrical stimulation cycling and electrical stimulation isometric exercise. Spinal Cord 2014;52:635-9.

51. Taylor MJ, Fornusek C, Ruys AJ. The duty cycle in Functional Electrical Stimulation research. Part II: Duty cycle multiplicity and domain reporting. Eur J Transl Myol 2018;28: In press.

52. Ranu EAS. Methods and systems for employing a duty cycle in electrical stimulation of patient tissue. US Patent 9,643,010 B2. May 9, 2017. Accessed via Google Patents, https://patents.google.com/ patent/US9643010B2/en?oq=USPTO+9643010.

53. Rigatelli G, Carraro U, Riccardi R, Rigatelli G. Demand dynamic biogirdling: ten-year results. J Thorac Cardiovasc Surg 2009;137:e58-9.

54. Rigatelli G, Rigatelli G, Barbiero $M$, et al. "Demand" stimulation of latissimus dorsi heart wrap: experience in humans and comparison with adynamic girdling. Ann Thorac Surg 2003;76:1587-92.

55. Carraro U, Rigatelli G, Rossini K, et al. Demand dynamic bio-girdling in heart failure: improved efficacy of dynamic cardiomyoplasty by LD contraction during aortic out-flow. Int $\mathrm{J}$ Artif Organs 2003;26:217-24.

56. Rigatelli G, Barbiero M, Rigatelli $\mathrm{G}$, et al. Maintained benefits and improved survival of dynamic cardiomyoplasty by activity-rest stimulation: 5-year results of the Italian trial on 
"demand" dynamic cardiomyoplasty. Eur J Cardiothorac Surg 2003;23:81-5.

57. Arpesella G, Carraro U, Mikus PM, et al. Activityrest stimulation of latissimus dorsi for cardiomyoplasty: 1-year results in sheep. Ann Thorac Surg 1998;66:1983-90.

58. Sajer S. Mobility disorders and pain, interrelations that need new research concepts and advanced clinical commitments. Eur J Transl Myol 2017;27(4):7179. doi: 10.4081/ejtm.2017.7179. eCollection 2017 Dec 5.

59. Carlson BM. The Biology of Long-Term Denervated Skeletal Muscle. Eur J Transl Myol 2014;24:3293. doi: 10.4081/ejtm.2014.3293. eCollection 2014 Mar 31.

60. Lomo T. The Response of Denervated Muscle to Long-Term Stimulation (1985, Revisited here in 2014). Eur J Transl Myol 2014;24:3294. doi: 10.4081/ejtm.2014.3294. eCollection 2014 Mar 31.

61. Carraro U, Kern H. Severely Atrophic Human Muscle Fibers With Nuclear Misplacement Survive Many Years of Permanent Denervation. Eur J Transl Myol 2016;26(2):5894. doi: 10.4081/ejtm.2016.5894. eCollection 2016 Jun 13.

62. Kern H, Boncompagni S, Rossini K, et al. Longterm denervation in humans causes degeneration of both contractile and excitation- contraction coupling apparatus, wich is reversibile by functional electrical stimulation (FES). A role for myofiber regeneration? J Neuropath Exp Neurol 2004;6:919-31.

63. Boncompagni S, Kern H, Rossini $\mathrm{K}$, et al. Structural differentiation of skeletal muscle fibers in the absence of innervation in humans. Proc Natl Acad Sci USA 2007;104:19339-44.

64. Kern H, Carraro U, Adami N, et al.. One year of home-based daily FES in complete lower motor neuron paraplegia: recovery of tetanic contractility drives the structural improvements of denervated muscle. Neurol Res 2010;32:26-3.

65. Kern H, Hofer C, Loefler Set al. Atrophy, ultrastructural disorders, severe atrophy and degeneration of denervated human muscle in SCI and Aging. Implications for their recovery by Functional Electrical Stimulation, updated 2017. Neurol Res 2017 http://dx.doi.org/10.1080/0161 6412.2017.1314906.

66. Carraro U, Kern H, Gava P, et al. Biology of Muscle Atrophy and of its Recovery by FES in Aging and Mobility Impairments: Roots and By-
Products. Eur J Transl Myol 2015;25(4):221-30. doi: 10.4081/ejtm.2015.5272. eCollection 2015 Aug 24.

67. Bijak M, Rakos M, Hofer C, et al. Stimulation parameter optimization for FES supported standing up and walking in SCI patients. Artif Organs. 2005 Mar;29(3):220-3.

68. Kern H, Carraro U. Home-Based Functional Electrical Stimulation for Long-Term Denervated Human Muscle: History, Basics, Results and Perspectives of the Vienna Rehabilitation Strategy. Eur J Transl Myol 2014;24(1):3296. doi: 10.4081/ejtm.2014.3296. eCollection Mar 31.

69. Kern H, Carraro U, Adami N, et al. Home-based functional electrical stimulation rescues permanently denervated muscles in paraplegic patients with complete lower motor neuron lesion. Neurorehabil Neural Repair 2010;24:709-21.

70. Carraro U, Edmunds KJ, Gargiulo P. 3D False Color Computed Tomography for Diagnosis and Follow-Up of Permanent Denervated Human Muscles Submitted to Home-Based Functional Electrical Stimulation. Eur J Transl Myol 2015;25(2):5133. doi: 10.4081/ejtm.2015.5133. eCollection 2015 Mar 11. Review.

71. Edmunds KJ, Gíslason MK, Arnadottir ID, et al.. Quantitative Computed Tomography and Image Analysis for Advanced Muscle Assessment. Eur J Transl Myol 2016;26(2):6015. doi: 10.4081/ejtm.2016.6015. eCollection 2016 Jun 13.

72. Edmunds K, Gíslason M, Sigurðsson S, et al. Advanced quantitative methods in correlating sarcopenic muscle degeneration with lower extremity function biometrics and comorbidities. PLoS One 2018;13(3):e0193241. doi: 10.1371/journal.pone.0193241. eCollection 2018.

73. Albertin G, Kern H, Hofer C, et al. Two years of Functional Electrical Stimulation by large surface electrodes for denervated muscles improve skin epidermis in SCI. Eur J Transl Myol 2018 Mar 6;28(1):7373. doi: 10.4081/ejtm.2018.7373. eCollection 2018 Jan 12.

74. Albertin G, Hofer Ch, Zampieri S, et al. In complete SCI patients, long-term functional electrical stimulation of permanent denervated muscles increases epidermis thickness. Neurol Res 2018 Apr;40(4):277-282. doi: 10.1080/01616412. 2018.1436877.

75. Brüggemann AK, Mello CL, Dal Pont $\mathrm{T}$, et al. Effects of Neuromuscular Electrical Stimulation 
During Hemodialysis on Peripheral Muscle Strength and Exercise Capacity: A Randomized Clinical Trial. Arch Phys Med Rehabil 2017 May;98(5):822-831.e1. doi: 10.1016/j.apmr. 2016.12.009. Epub 2017 Jan 16.

76. McGregor G, Ennis S, Powell R, et al. Feasibility and effects of intra-dialytic low-frequency electrical muscle stimulation and cycle training: A pilot randomized controlled trial. PLoS One. $2018 \mathrm{Jul}$ 11;13(7):e0200354. doi: 10.1371/journal.pone. 0200354. eCollection 2018.

77. Jones S, Man WD, Gao W, et al. Neuromuscular electrical stimulation for muscle weakness in adults with advanced disease. Cochrane Database Syst Rev. 2016 Oct 17;10:CD009419.

78. Mosole S, Carraro U, Kern H, et al. Use it or Lose It: Tonic Activity of Slow Motoneurons Promotes Their Survival and Preferentially Increases Slow Fiber-Type Groupings in Muscles of Old Lifelong Recreational Sportsmen. Eur J Transl Myol. 2016 Nov 25;26(4):5972. doi: 10.4081/ejtm.2016.5972. eCollection 2016 Sep 15.

79. Mosole S, Carraro U, Kern H, et al. Long-term high level exercise promotes muscle reinnervation with age. J Neuropathol Exp Neurol. 2014 Apr;73(4):284-94.

doi: 10.1097/NEN.0000000000000032.

80. Power GA, Dalton BH, Gilmore KJ, et al. Maintaining Motor Units into Old Age: Running the Final Common Pathway. Eur J Transl Myol 2017;27(1):6597. doi: 10.4081/ejtm.2017.6597. eCollection 2017 Feb 24. -80

81. Willand MP. Electrical Stimulation Enhances Reinnervation After Nerve Injury. Eur J Transl Myol 2015;25(4):243-8. doi: 10.4081/ejtm. 2015.5243. eCollection 2015 Aug 24. Review.

82. Carraro U, Kern H, Gava P, et al. Recovery from muscle weakness by exercise and FES: lessons from Masters, active or sedentary seniors and SCI patients. Aging Clin Exp Res. 2017 Aug;29(4):579590. doi: 10.1007/s40520-016-0619-1. Epub 2016 Sep 3. Review.

83. Carraro U, Gava K, Musumeci A, et al. Safe Antiaging Full-Body In-Bed Gym and FES for Lazy Persons: Home In-Bed Exercises for Fighting Muscle Weakness in Advanced Age. In: Rehabilitation Medicine for Elderly Patients, Masiero S, Carraro U, Eds., 2018, pag. 43-52. ISBN 978-3-319-57405-9 ISBN 978-3-319-574066 (eBook).
84. Carraro U, Albertin G, Gargiulo P, et al. Muscle and skin improve by home-based FES and fullbody in-bed gym. Biol Eng Med, 2018;3(3):1-42018. doi: 10.15761/BEM.1000S1003.

85. "Stimulette den2x" Available at: https://www. schuhfried.com/umbraco/Surface/AuthenticationSu rface/Login?return Url=\%2Fportal.

86. Ekelem A, Goldfarb M. Supplemental stimulation improves swing phase kinematics during exoskeleton assisted gait of SCI subjects with severe muscle spasticity. Front Neurosci 2018 Jun 1;12:374. doi: 10.3389/fnins.2018.00374. eCollection 2018.

87. Angeli CA, Boakye M, Morton RA, et al. Recovery of Over-Ground Walking after Chronic Motor Complete Spinal Cord Injury. N Engl J Med 2018;379(13):1244-50. doi: 10.1056/NEJMoa 1803588. Epub 2018 Sep 24.

88. Harkema SJ, Legg Ditterline B, Wang S, et al. Epidural Spinal Cord Stimulation Training and Sustained Recovery of Cardiovascular Function in Individuals With Chronic Cervical Spinal Cord Injury. JAMA Neurol 2018 Sep 17. doi: 10.1001/jamaneurol.2018.2617. [Epub ahead of print].

89. Herrity AN, Williams CS, Angeli CA, et al. Lumbosacral spinal cord epidural stimulation improves voiding function after human spinal cord injury. Sci Rep 2018 Jun 6;8(1):8688. doi: 10.1038/s41598-018-26602-2.

90. Aslan SC, Legg Ditterline BE, Park MC, et al. Epidural Spinal Cord Stimulation of Lumbosacral Networks Modulates Arterial Blood Pressure in Individuals With Spinal Cord Injury-Induced Cardiovascular Deficits. Front Physiol 2018;9:565. doi: 10.3389/fphys.2018.00565. eCollection 2018.

91. Harkema SJ, Wang S, Angeli CA, et al. Normalization of Blood Pressure With Spinal Cord Epidural Stimulation After Severe Spinal Cord Injury. Front Hum Neurosci 2018;12:83. doi: 10.3389/fnhum.2018.00083. eCollection 2018.

92. Terson de Paleville DGL, Harkema SJ, Angeli CA. Epidural stimulation with locomotor training improves body composition in individuals with cervical or upper thoracic motor complete spinal cord injury: A series of case studies. J Spinal Cord Med 2018 Mar 14:1-7.doi: 10.1080/10790268. 2018.1449373. [Epub ahead of print]

93. Hubscher $\mathrm{CH}$, Herrity AN, Williams CS, et al. Improvements in bladder, bowel and sexual 
outcomes following task-specific locomotor training in human spinal cord injury. PLoS One 2018;13(1):e0190998. doi: 10.1371/journal.pone. 0190998. eCollection 2018.

94. Wagner FB, Mignardot JB, Le Goff-Mignardot CG, et al. Targeted neurotechnology restores walking in humans with spinal cord injury. Nature 2018 Nov;563(7729):65-71. doi: 10.1038/s41586-0180649-2. Epub 2018 Oct 31.

95. Formento E, Minassian K, Wagner F, et al. Electrical spinal cord stimulation must preserve proprioception to enable locomotion in humans with spinal cord injury. Nat Neurosci 2018 Oct 31. doi: 10.1038/s41593-018-0262-6. [Epub ahead of print]

96. Capogrosso M, Wagner FB, Gandar J, et al. Configuration of electrical spinal cord stimulation through real-time processing of gait kinematics. Nat Protoc 2018Sep;13:2031-61. doi: 10.1038/s41596018-0030-9.
97. Capogrosso M, Gandar J, Greiner N, et al. Advantages of soft subdural implants for the delivery of electrochemical neuromodulation therapies to the spinal cord. J Neural Eng 2018;15:026024. doi: 10.1088/1741-2552/aaa87a. 10.1088/1741-2552/aaa87a.

98. Moraud EM, von Zitzewitz J, Miehlbradt J, et al. Closed-loop control of trunk posture improves locomotion through the regulation of leg proprioceptive feedback after spinal cord injury. Sci Rep 2018;8:76. doi: 10.1038/s41598-017-18293-y.

Received for publication: 02/8/2018

Revision received: 20/9/2018

Accepted for publication: 21/9/2018 